Results The main pain reported by the staff was lumbar pain (70\%).

Several factors explain that result:

- Repeatedly carrying heavy weights (>7 Kg), especially when loading the Instrument Washer-Disinfector trolleys and sterilisers.

- Making little use of helping fork-lift trucks ( $60 \%$ of the staff use them $<2$ hrs/day).

- Not asking colleagues for help when carrying heavy weights.

- $80 \%$ of people work in front of a computer screen for $1 / 3$ or $1 / 2$ the day without adopting an ergonomic position.

- Highly repetitive actions during packaging.

Preventive measures:

- Staff training on ergonomics suited to any post.

- Organization of packaging posts and data capture according to the "comfort zone" concept.

- Reduction of distances to be covered when carrying or moving heavy weights.

Conclusions This study demonstrates that MSDs often appeared in sterilisation. The implementation of suitable preventive measures - according to posts - should increase efficiency and reduce the physical demands made on members of staff.

No conflict of interest.

\section{GRP-018 ANALYSIS OF ANTINEOPLASTIC MEDICATION ERRORS IN A 500-BED TEACHING HOSPITAL}

doi:10.1136/ejhpharm-2013-000276.018

R Gavira Moreno, MT Moreno Carvajal, V González Rosa, F Gómez de Rueda, R Gázquez Pérez, JP Díaz López, P Gómez Germá, MA Almendral Vicente, MT Gómez de Travecedo y Calvo, M Lobato Ballesteros. Hospital de/ SAS de Jerez, Farmacia, Jerez de la Frontera (Cádiz), Spain

Background Medication errors with antineoplastic drugs may be catastrophic due to the drugs' high toxicity and narrow therapeutic index.

Purpose To assess antineoplastic medication errors in terms of frequency, type of error and severity for patients.

Materials and Methods A 1-year prospective study was conducted (2011) in order to identify the medication errors that occurred during cancer chemotherapy for patients in a 500-bed teaching hospital. Wards included both day care and inpatient units. All prescriptions and production forms were verified by pharmacists. The different types of error were defined in a data collection form. For each medication error intercepted, the potential severity was evaluated according to the Ruiz-Jarabo 2000 version2 classification system.

Results During the study period, the pharmacy unit prepared 17241 distinct anticancer drugs. In total, 136 medications errors were detected throughout the medicines use process. Prescriptions errors represented $82 \%$ of errors, followed by pharmaceutical validation $(7 \%)$ transcription $(7 \%)$, preparation $(2 \%)$ and administration errors (2\%).

The most common causal drug was carboplatin, which was involved in 25 cases, despite corresponding to only $2.8 \%$ of anticancer drugs prescribed at our institution. Overall, in 66 cases erroneous doses of the medicine were recorded (48.5\%), 24 errors were linked to the choice of antineoplastic regimen $(17.6 \%)$ while in 12 cases, erroneous duration of treatment was prescribed (8.8\%).

Of the 136 medication errors, 124 were intercepted prior to administration while 12 reached the patients (9\%). Overall $66 \%$ of non-intercepted medication errors had no impact on the patient and only 3 cases required enhanced monitoring.

Conclusions In our study pharmaceutical validation mainly allowed us to identify prescription errors (82\%), almost all errors were intercepted prior to administration to the patient. Wrong dose represented the most common type of error. Few pharmaceutical errors (transcription, validation, preparation) were detected.

No conflict of interest.

\section{GRP-019 ANALYSIS OF ANTIRETROVIRAL TREATMENT ADHERENCE IN OUTPATIENTS OVER A TWO-YEAR PERIOD OF STUDY}

doi:10.1136/ejhpharm-2013-000276.019

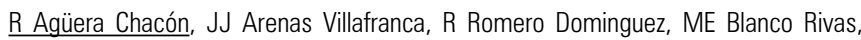
C Lopez Martín, M Garrido Siles, B Tortajada Goitia, V Faus Felipe. Hospital Costa del Sol, Farmacia y Nutrición, Marbella, Spain

Background The efficacy and safety of anti-retroviral treatment is affected by many factors and compliance is key in therapy success. A lack of adherence may lead to therapeutic failure and higher rates of drug resistance.

Purpose To describe collected data about outpatient antiretroviral treatment adherence and analyse characteristics and factors associated with the non-adherent population.

Materials and Methods A retrospective observational study was conducted over 27 months on all outpatients on antiretroviral therapy who attended our hospital for human immunodeficiency virus (HIV) monitoring between June 2010 and September 2012. Each patient's adherence was checked and recorded every 6 months. This was measured as '(Total no. of units dispensed/Total no. of units needed) $\times 100$ '. Those patient with adherence $>95 \%$ were considered as 'adherent' and those with $<95 \%$ as 'non-adherent'. All results were recorded in a database. For the 'non-adherent' population the following features were reviewed: Sex, age, drug use, presence of Hepatitis B (HBV) or Hepatitis C (HCV) and total number of tablets/day, including drugs for other diseases besides HIV

Results During the period of study, 1841 adherence cheques were made on a total of 630 patients (2.9 tests/patient). $24.6 \%$ of the HIV patients in treatment were non-adherent in at least one cheque. Their average age was $45.5 \pm 8.6$ years, $74 \%$ men, mean treatment duration of $8 \pm 4.4$ years, and a median consumption per day of 4 doses (range 1 to 16 ). $35.5 \%$ of these patients took drugs, $7.1 \%$ were co-infected with HBV and $45.2 \%$ were co-infected with HCV $(5.2 \%$ was co-infected with both viruses). The Chi-square test showed a significant relationship $(p<0.05)$ between substance abuse, HCV infection and male gender in non-adherent patients.

Conclusions The study revealed a large percentage of nonadherent patients who compromised the effectiveness of their antiretroviral treatment. The intervention of hospital pharmacists, checking on compliance and following up with patients, could play an important role in reducing this negative factor, especially in those with HCV and/or substance abuse.

No conflict of interest.

\section{GRP-020 ANALYSIS OF ITALIAN HOSPITAL PHARMACIST ACTIVITIES TO PREVENT LASA DRUG ERRORS IN TREATMENT: FIRST RESULTS}

doi:10.1136/ejhpharm-2013-000276.020

${ }^{1} \mathrm{~N}$ Ciociano, ${ }^{2} \mathrm{~F}$ Saullo, ${ }^{1} \mathrm{M}$ Pacillo, ${ }^{1} \mathrm{~F}$ Romano, ${ }^{3} \mathrm{~L}$ Bagnasco. ${ }^{1}$ Università degli Studi di Salerno, Scuola di Specializzazione in Farmacia Ospedaliera, Fisciano (Sa), Italy; ${ }^{2}$ Azienda Ospedaliera Mater Domini, Centro Regionale di Documentazione ed Informazione sul Farmaco, Catanzaro, Italy; ${ }^{3}$ ASL Cuneo 1, Farmacia Ospedaliera, Saluzzo (Cn), Italy

Background Errors caused by the use of Look-Alike/Sound-Alike (LASA) drugs occur with high frequency in hospital departments. In August 2010 the Italian Ministry of Health passed a Recommendation to help health operators to reduce LASA errors, through 
special procedures of clinical management. After two years, an independent study seeking to explore the awareness of this Recommendation and its implementation by Italian hospital pharmacists has started. It is designed in two steps that differ for methodology of enrolment: in step 1 only Directors of pharmacy departments are enrolled; in step 2 all hospital pharmacists working in Health National System hospitals will be enrolled.

Purpose To describe the results of step 1.

Materials and Methods In the period 01/08/2012-30/09/2012, 250 Directors of Italian pharmacy departments were enrolled. They received a questionnaire composed of 11 questions on the following topics: knowledge of LASA drugs and the ministerial Recommendation; any LASA drug errors and causes detected in their hospital in the period August 2010-August 2012; activation of risk management procedures to prevent LASA and implement the Recommendation in their hospital.

Results $52.5 \%$ of Pharmacists answered: $100 \%$ were familiar with LASA drugs and the ministry Recommendation. $73 \%$ had detected LASA drug errors in their hospital, caused by the following similarities: $66 \%$ packaging; $14 \%$ trade name, $6 \%$ active substance name, $6 \%$ association brand name and packaging; $8 \%$ association active substance name and packaging. $58 \%$ had publicised the Recommendation in their hospital but only $22 \%$ had adopted specific measures of risk management.

Conclusions The results could reflect little interest in preventing LASA errors by enrolled pharmacists. It is an alarming situation. If step 2 confirms this trend, it will be necessary to implement a new Ministerial Intervention against LASA drug errors in Italy.

No conflict of interest.

\section{GRP-021 ANALYSIS OF PHARMACIST INTERVENTIONS DURING THE VALIDATION OF THE ELECTRONIC PRESCRIPTIONS IN A SPANISH HOSPITAL}

doi:10.1136/ejhpharm-2013-000276.021

'F Caracuel, ${ }^{1} \mathrm{C}$ Tournus, 'U Baños, 'ML Moya, 'M Beltrán, ' $\mathrm{G}$ Ramírez. ${ }^{1}$ Hospital Universitario Virgen Macarena, Pharmacy, Seville, Spain; ${ }^{2}$ Université de Lyon, Pharmacy, Lyon, France

Background Computerized provider-order-entry (CPOE) system is known to improve quality, increase efficiency, and reduce medication errors.

The pharmacist, through the electronic validation, can provide improvements to the patient pharmacotherapy. However, not all hospitals follow the same method to make such proposals.

Purpose To analyse the type of interventions made in our hospital.

To validate process intervention.

Materials and Methods Pharmacists interventions were studied over a period of one year (June 2011-May 2012). Both prescription and validation are performed in the computer programme Farmatools ${ }^{\circledR}$. The pharmacist used to write a warning on the patient treatment. Alerts were reviewed the following day and we checked if the recommendation was accepted or not by the physician. Interventions were classified according to the type of recommendation, the drug and whether it was accepted.

Results A total of 788 interventions were analysed ( 2.2 per day). The most frequent (27\%) was dose adjustment for renal failure, followed by switching from intravenous to oral route $(16 \%)$, change of dose $(13 \%)$ and indication $(12 \%)$. Other interventions were medication reconciliation, duplicity, therapeutic equivalent and adverse reaction.

The most frequent drugs were enoxaparin (24\%), pantoprazole $(12 \%)$, paracetamol $(5 \%)$, insulin $(5 \%)$, digoxin (4\%), amoxicilinclavulanic (4\%) and levofloxacin (4\%).

Only $72 \%$ of the recommendations were reviewed. From this, $54 \%$ were accepted.
Conclusions Although 788 interventions have been studied, there are many who have not been registered in the programme, so it could not be analysed. We observed that the dose adjustment for renal failure, especially enoxaparin, is recorded systematically, but this does not occur with other types of interventions.

Acceptance is lower than those reported in literature, so we can conclude that the method of communication with the clinician is inadequate and should be strengthened with verbal communication.

No conflict of interest.

\section{GRP-022 ANALYSIS OF THE MEDICINES RECONCILIATION PROCESS IN DIFFERENT CLINICAL SERVICES}

doi:10.1136/ejhpharm-2013-000276.022

L Corrales Pérez, B Rubio Cebrián, I Gasanz Garicochea, M Segura Bedmar, C Calderón Acedos, MJ Vázquez Castillo, C Moriel Sánchez, P San Miguel Torvisco, R Catalá Pizarro. Hospital Universitario de Móstoles, Farmacia Hospitalaria, Madrid, Spain

Background Medication errors, specifically the lack of continuity of the patient's usual treatment, are a major cause of adverse effects in hospitalised patients, most of them preventable. Medicines reconciliation is the process of comparing a patient's prescriptions for medicines to all the medicines the patient has been taking.

Purpose To analyse the impact of reconciliation in different clinical services depending on discrepancies identified and severity of medicines errors (MEs)

Materials and Methods Retrospective, descriptive study conducted at a general hospital over 6 months. Daily, we identified newly-hospitalised patients aged over 75 . To determine that a discrepancy existed, we compared the patient's usual medicines with the prescribed medicines and interviewed patient and/or carers. For each service, we collected: number of patients reconciled, number of drugs evaluated, kinds of discrepancies according to Documento de consenso sobre terminología, clasificación y evaluación de los programas de Conciliación de la Medicación, and severity of MEs identified according to National Coordinating Council for Medication Error Reporting and Prevention.

Results Reconciliation was conducted in 13 clinical services. 558 patients were reconciled (mean age: 83.86). 56\% belonged to Internal Medicine (IM), followed by General Surgery (GS) (18\%) and Traumatology (13\%). 9.33 drugs were evaluated per patient, higher than average numbers of prescribed drugs being found in Ophthalmology (18), Cardiology (17.48), IM (11.62), Pneumology (11.29) and Oncology (10.38). We detected 1140 discrepancies. The services with more discrepancies requiring clarification $(n=412)$ were: IM (51\%), GS (16\%) and Traumatology (12\%). The services with the highest rates of MEs were Traumatology $(60 \%)$, Otolaryngology (60\%), Pneumology (59\%), Urology (57\%) and Haematology $(50 \%)$, while unresolved discrepancies were noted in Gynaecology (78\%), Oncology (64\%), GS (51\%) and Ophthalmology (50\%). Most MEs fell into category C (errors that reached patient but did not cause damage) severity but $1 \%$ were category $\mathrm{E}$ (error that resulted in temporary harm and required an intervention). The omission of a medicine was the most common unjustified discrepancy.

Conclusions Medicines reconciliation is important in IM, GS and Traumatology because of numerous discrepancies requiring clarification, the proportion of patients and, mainly in IM, the amount of drugs for chronic treatment. The role of reconciliation was judged essential in clinical services with more MEs (Traumatology, Otolaryngology). Unresolved discrepancies pose a potential cause of $\mathrm{ME}$, so in Gynaecology and Oncology we should improve communication with clinical teams to encourage patient safety.

No conflict of interest. 